\title{
La charte européenne de la coopération en matière d'appui à la gouvernance locale : outil et questionnements pour les autorités territoriales
}

The European Charter of Cooperation in Terms of Support for Local Governance: Instrument and Concerns for Territorial Authorities

\section{Gaëlle Dusepulchre}

\section{OpenEdition \\ Journals}

Édition électronique

URL : http://journals.openedition.org/developpementdurable/8380

DOI : 10.4000/developpementdurable.8380

ISSN : 1772-9971

Éditeur

Association DD\&T

Référence électronique

Gaëlle Dusepulchre, «La charte européenne de la coopération en matière d'appui à la gouvernance locale : outil et questionnements pour les autorités territoriales », Développement durable et territoires [En ligne], Vol. 1, n 1 | Mai 2010, mis en ligne le 01 octobre 2010, consulté le 30 avril 2019. URL: http://journals.openedition.org/developpementdurable/8380; DOI : 10.4000/ developpementdurable.8380

Ce document a été généré automatiquement le 30 avril 2019.

Développement Durable et Territoires est mis à disposition selon les termes de la licence Creative Commons Attribution - Pas d'Utilisation Commerciale 4.0 International. 


\section{La charte européenne de la coopération en matière d'appui à la gouvernance locale : outil et questionnements pour les autorités territoriales}

The European Charter of Cooperation in Terms of Support for Local Governance: Instrument and Concerns for Territorial Authorities

\section{Gaëlle Dusepulchre}

1 Un consensus tend à émerger sur l'intérêt, pour les autorités locales et régionales (ALR), de s'investir dans des activités de coopération au développement, lesquelles répondraient aux impératifs de globalisation et de solidarité, et offriraient à ces acteurs un outil de cohésion sociale, de croissance économique et d'innovation (UN Habitat, 2006:9-11; Gaviria, A.A, $2005: 74$; Desmet A., Develtere P., 2002:6-8; Comité des Régions, 2004 ; Local government Association).

2 Parallèlement, les bailleurs traditionnels tendent à encourager, et à reconnaître la plus value potentielle, de l'investissement relativement récent des villes et régions dans des activités de coopération au développement. En effet, distinguées aujourd'hui des autres acteurs de la coopération, les ALR, en qualité de pouvoirs publics de proximité légitimes, sont à présent perçues comme susceptibles de créditer les activités de coopération au développement de l'expertise qu'elles ont acquise dans la gestion de leurs propres affaires publiques et, partant, de relayer les bailleurs traditionnels et de combler leurs lacunes en ces domaines (Commission européenne, 2008a : 3-4 ; Commission européenne, 2008b : 2 ; OCDE, 2005 : 13 ; UN Habitat, 2006 : 5).

3 Pour autant, l'UE, qui s'est engagée à soutenir l'investissement croissant des ALR européennes dans les activités de coopération, souligne la nécessité de pallier le risque de fragmentation de l'assistance au développement que pourrait induire l'implication 
grandissante de ces nouveaux acteurs. Elle insiste sur l'importance que les ALR renoncent à s'inscrire en marge des efforts internationaux entrepris pour accroître l'efficacité de l'aide en faisant valoir « leurs compétences et prérogatives propres ». Elle se propose, en conséquence, d'encourager toute initiative qui participerait à structurer davantage leur intervention (Conseil UE, 2008, § 4 ; Commission européenne, 2008b : 9).

Les institutions européennes, diverses ALR européennes et leurs réseaux d'associations s'entendraient alors sur la nécessité de centraliser les informations, de définir les bonnes pratiques, et de coordonner les appuis apportés aux États en développement (Conseil UE, 2008, § 4; Commission européenne, 2008b : 9 ; Commission européenne 2008c : 14 ; CCRE, 2008a, \$ 4 ; CCRE, 2008b; CGLU, 2008 ; Metropolis). La charte européenne de la coopération en matière d'appui à la gouvernance locale, fruit d'une initiative d'ALRs françaises, relayée par la présidence de l'Union et à laquelle s'est associée la Commission européenne notamment, serait l'une des premières illustrations de cette nouvelle dynamique. Officialisée en décembre 2008, il s'agit du premier texte européen qui s'attèle à renforcer l'efficacité, la cohérence et la coordination des activités de coopération au développement conduites en appui aux pouvoirs locaux dans les pays partenaires. La charte réaffirme les principes dégagés sur la scène internationale en 2005 et consacrés par la Déclaration de Paris. Elle consacre, en outre, les grands axes d'intervention et les précautions à prendre pour que les appuis s'inscrivent dans l'effort global pour la gouvernance. Le Comité des régions et les associations d'ALR qui ont participé à son élaboration (aux côtés de la majorité des États membres et de la Commission européenne notamment) insistent sur la pertinence et l'importance de ce texte, de même que le conseil de l'UE, qui suggère qu'elle joue un rôle directeur dans les appuis fournis par les ALR européennes (Charte 2008; Conseil UE, 2008, §8-11; Commission européenne, 2008b, pp 2, 3; Commission européenne, 2008c:14; Commission européenne 2008d; ECDPM, 2008; CNCD 2008; Comité des Régions, 2009 ; CGLU, 2008 ; CCRE, 2008a) ${ }^{1}$.

5 Le présent article tend à souligner la plus value de la charte s'agissant de clarifier le concept de gouvernance à l'adresse des nouveaux acteurs décentralisés du développement (1), autant qu'il vise à mettre en exergue certains des questionnements sur lesquels les acteurs devront à terme se positionner (2).

\section{Un nouvel outil à l'appui des stratégies de coopération au développement qui promeuvent la gouvernance}

6 La charte tend à assurer que les appuis portés aux pouvoirs locaux élus dans les pays partenaires (contre-pouvoirs décentralisés) participent au modèle de gouvernance qui a été élaboré par les bailleurs traditionnels comme la Banque Mondiale, l'Union européenne et le PNUD à l'appui de leurs stratégies de coopération (UNDP 2004, 2006 , 2007; World Bank 1992, 1994, 1997, 2000, 2002, 2003, 2007, 2007b, 2007c; Conseil UE 1998, Conseil UE , 2003 ; UE, 2000 ; UE, 2005 ; Commission européenne1993, 2000, 2003, 2005, 2006). 


\subsection{Le modèle de gouvernance promu dans les stratégies adressées aux États en développement}

7 Ce modèle, promu notamment par l'Union européenne et la Banque Mondiale, repose principalement sur trois principes directeurs : transparence, empowerment et accountability (AET) (World Bank 1994, 1997, 2000, 2002, 2003, 2007 ; Conseil UE 1998, Conseil UE, 2003 ; UE, 2000 ; UE, 2005 ; Commission européenne 1993, 2000, 2003, 2005, 2006).

8 La doctrine véhiculée par ces institutions internationales postule que ces principes sont supposés contribuer à la qualité de la conception des politiques publiques, de leur mise en œuvre, de leur suivi (effectivité) et de leur évaluation (pertinence) et commanderont, le cas échéant, des réformes structurelles (pour une analyse complète ainsi que des références questionnant la pertinence et les limites de leurs fondements théoriques voir Dusepulchre G., 2009).

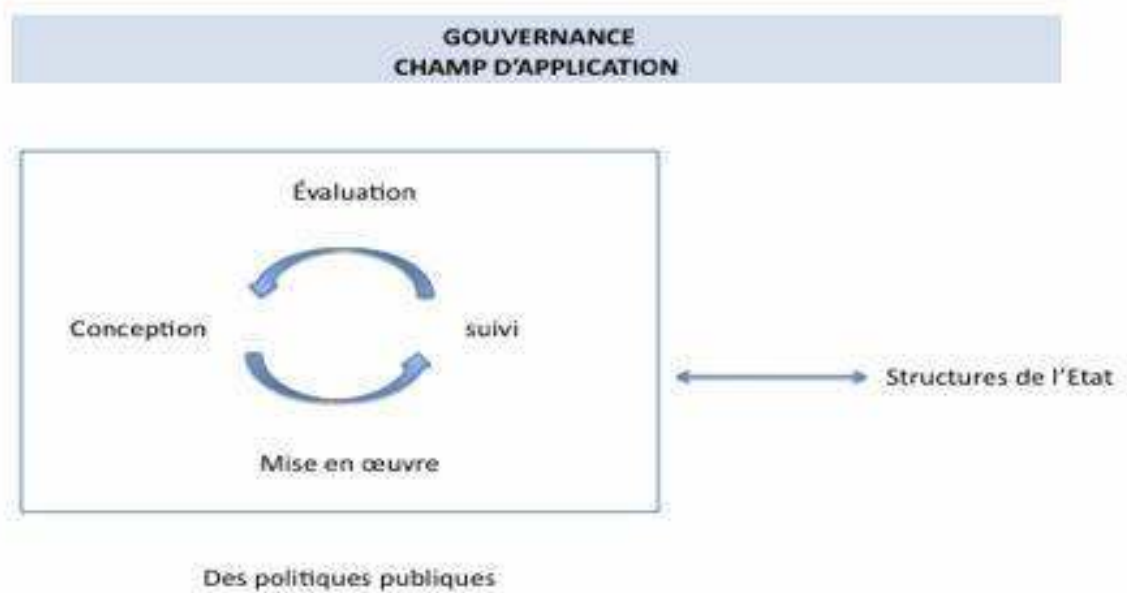

9 La transparence suppose de réunir les conditions utiles à une stratégie équilibrée, à savoir information adéquate, motivation et publicité. L'accountability vise la reddition de comptes. Elle suppose d'instituer les contrôles utiles à la bonne conduite des affaires publiques. Enfin l'empowerment suppose de garantir les capacités utiles à la gestion publique (règles, structures, procédures, moyens humains, techniques et financiers). Ces principes sont interdépendants et se renforcent mutuellement. 


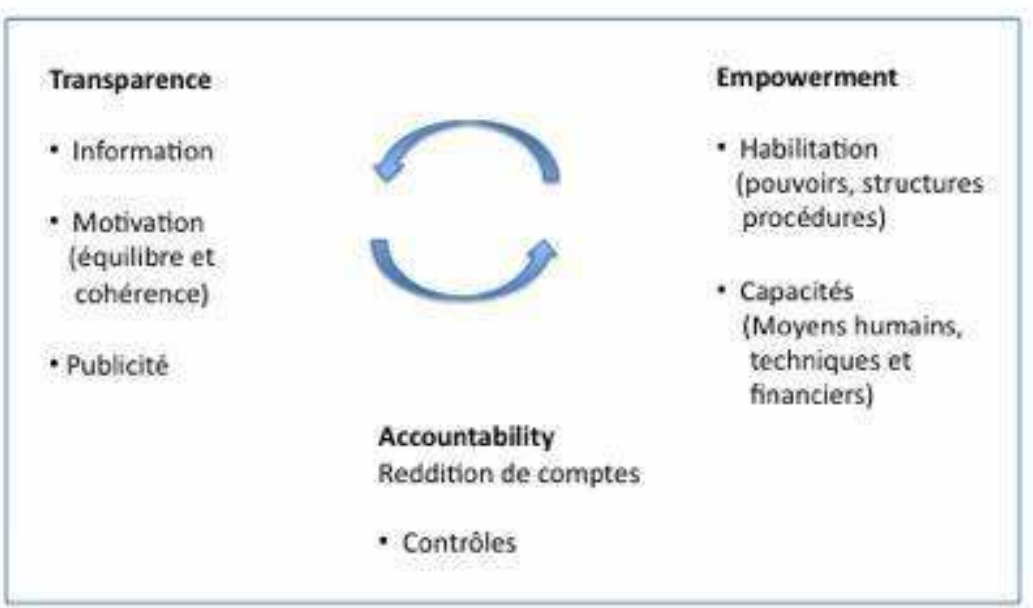

Les bailleurs postulent, en outre, que la bonne gouvernance est le fruit d'un équilibre entre les intérêts concurrents des diverses composantes de la société, et que cet équilibre ne peut être atteint que s'il existe des contre-pouvoirs c'est-à-dire une compétition d'acteurs intéressés par la gestion de la chose publique et en mesure d'interagir avec elle. Transposant cet entendement de la gouvernance à la sphère politique et économique, les bailleurs plaideront à ce titre la décentralisation, la séparation des pouvoirs, la participation de la population à la gestion de l'État, la concurrence dans la délivrance des services publics, la privatisation, la dérégulation, l'économie de marché, et le partenariat public-privé.

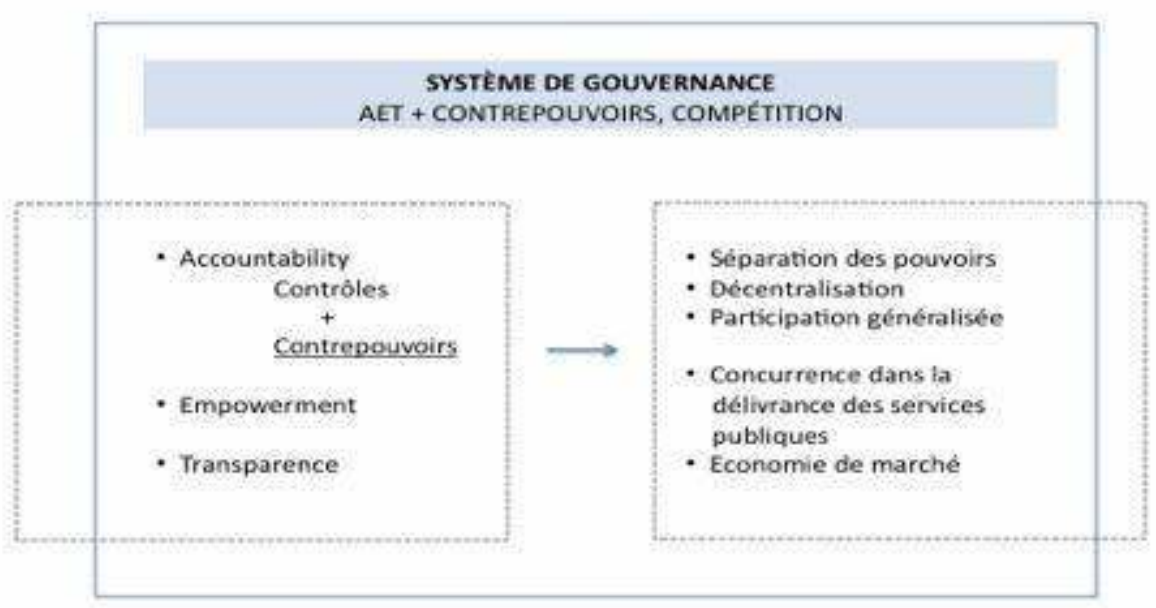

11 Les bailleurs font de l'existence de ces contre-pouvoirs une garantie de la reddition de compte (accountability). Dans ce cadre, le contrôle ne pourra s'entendre comme simplement hiérarchisé et aux mains du seul pouvoir exécutif, mais davantage comme reposant sur l'activité des contre-pouvoirs (autorités décentralisées, sociétés civiles, 
acteurs sociaux et économiques). La transparence supposera d'associer ces contrepouvoirs à la conception des politiques publiques, ainsi qu'à leur mise en œuvre, leur suivi et leur évaluation. L'empowerment, pour sa part, commandera de les doter des moyens et capacités utiles à leur participation (y compris les droits et les libertés civiles et politiques par exemple). La charte participe à ce modèle, tant en ce qu'elle promeut un soutien accru aux contrepouvoirs décentralisés des États en développement, qu'en ce qu'elle défini les axes d'interventions supposés en assurer la viabilité (1.2).

\subsection{Transposition aux contextes de décentralisation : les appuis utiles à la gouvernance locale} «bonne gouvernance» au motif que les autorités décentralisées seraient le contrepouvoir idéal à l'État central, parce que, plus proches des besoins et des réalités locales, elles seraient plus efficaces pour remplir certaines fonctions (Charte 2008:3; UNDP, 2004). Toutefois l'expérience acquise en matière de décentralisation dans les États en développement a révélé que les autorités locales ne participent pas nécessairement à l'émergence d'une gouvernance locale participative, qu'elles ne sont pas par essence «pro pauvres», et qu'elles ne sont pas forcément plus efficientes dans la gestion des affaires publiques (Comité des Régions, 2009b, §16; Europaid, $2007: 2$; UNDP $2007: 6-26$ ). Ces constats ont conduit les bailleurs à imputer les failles des processus de décentralisation à une conception non conforme ou une mise en œuvre insuffisante de ses principes fondateurs. En conséquence, le postulat selon lequel certaines affaires sont mieux gérées au niveau infra étatique, est aujourd'hui présenté comme largement tributaire de la capacité des entités décentralisées à s'inscrire dans un contexte de gouvernance (UNDP 2007 : 6 ; UNDP 2006, UN Habitat, 2005, pt. 2). Quatre axes d'intervention ont été dégagés à cet effet par la charte européenne de la coopération en matière d'appui à la gouvernance locale.

\section{Promouvoir un cadre favorable à la gouvernance locale démocratique}

Le premier axe d'intervention décrit par la charte vise, d'une part, à renforcer le dialogue entre le pouvoir central et les pouvoirs locaux des pays partenaires, d'autre part, le développement des capacités, tant des autorités locales et de leurs réseaux associatifs, que des structures centrales en charge des politiques de décentralisation. Ces appuis sont supposés contribuer à l'élaboration de stratégies cohérentes et équilibrées, ainsi qu'à l'institution des mesures de suivi et d'évaluation qui garantissent l'effectivité des processus de décentralisation. 


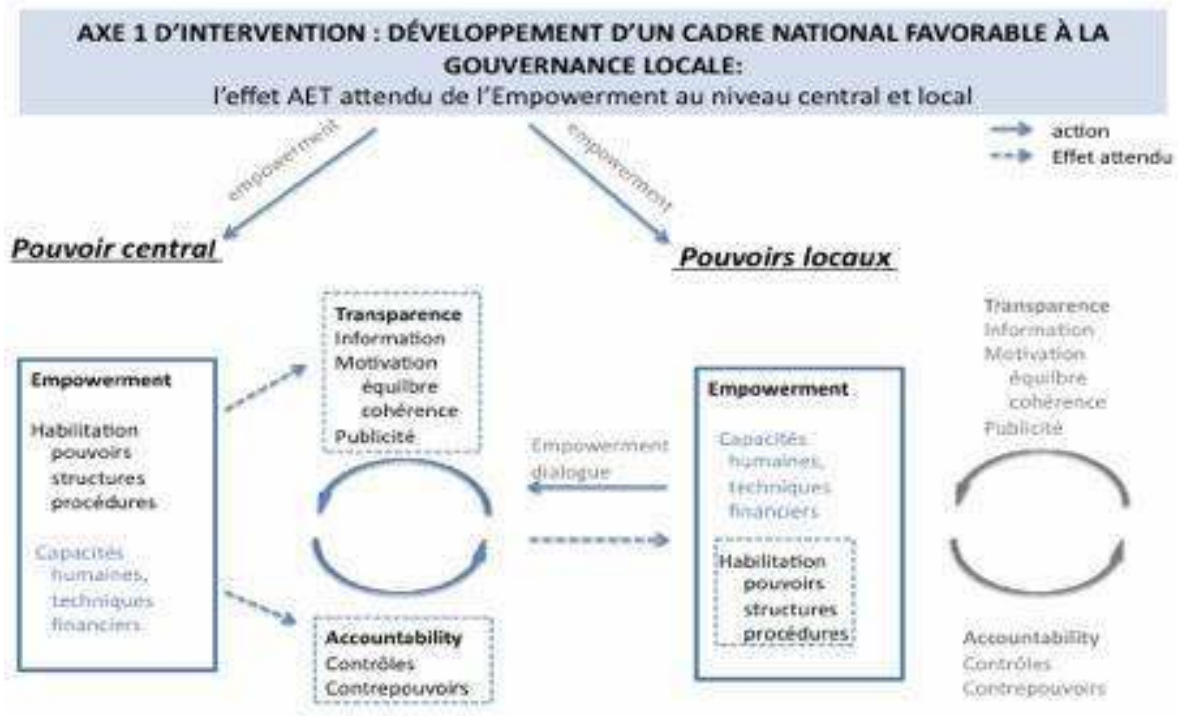

La charte propose également de renforcer les moyens dont disposent les ALR pour se faire entendre et d'accroître leur poids dans le dialogue. Elle encourage, à cette fin, l'appui au développement de réseaux associatifs nationaux et transnationaux d'une part et à l'instauration d'un canal de communication privilégié avec les institutions centrales, en charge des processus de décentralisation, et donc davantage sensibles à la question, d'autre part (Europaid, $2007: 2$; Miossec J-M, 2008 : 59-61).

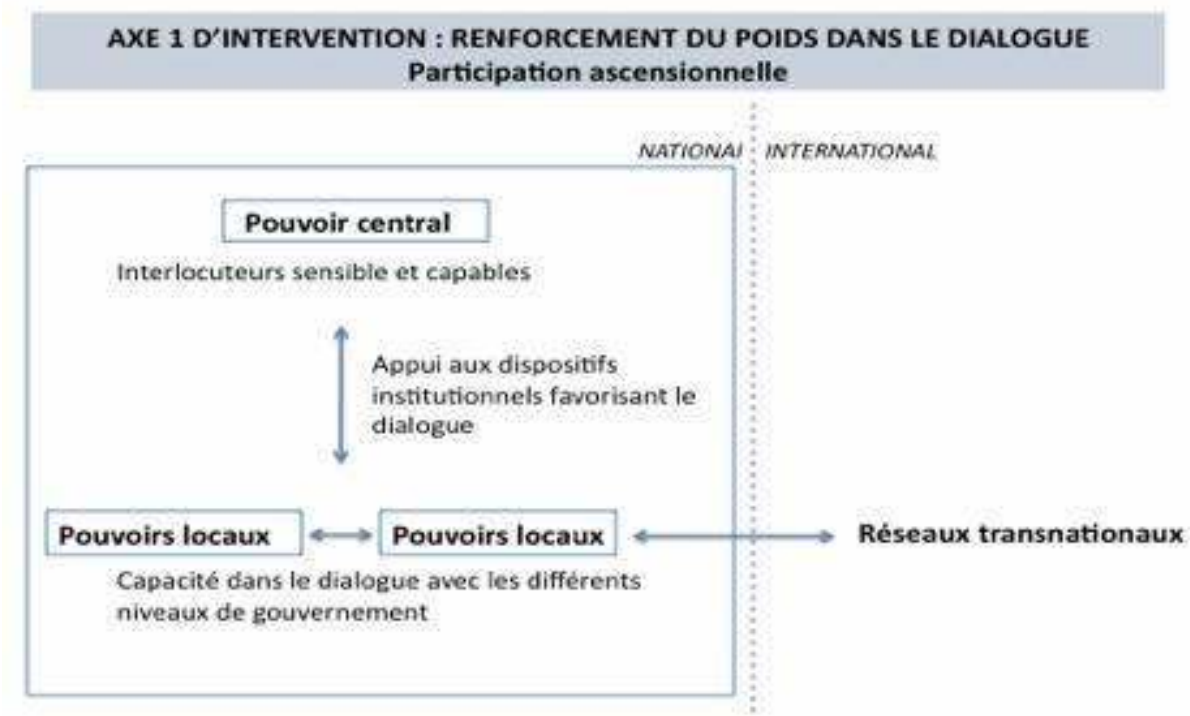

\section{Renforcer la gouvernance démocratique à l'échelle locale}

Le second axe d'intervention en appui à la gouvernance locale s'intéresse aux mécanismes d'accountability ascendants (vers le gouvernement central), descendants (vers les citoyens) et horizontaux (au sein des entités décentralisées). Il insiste tout particulièrement sur les mécanismes descendants et le rôle des populations comme contre-pouvoir et acteurs du développement. Ainsi, les pouvoirs locaux devront êtres 
organisés et disposer des capacités utiles à conduire des politiques participatives emportant le consensus de l'ensemble des groupes sociaux (même marginalisés). En outre, "l'implication des organisations de la société civile dans l'élaboration, la planification, la budgétisation et le suivi-évaluation des politiques » supposera également un droit à l'information, et le développement des capacités de ces populations.

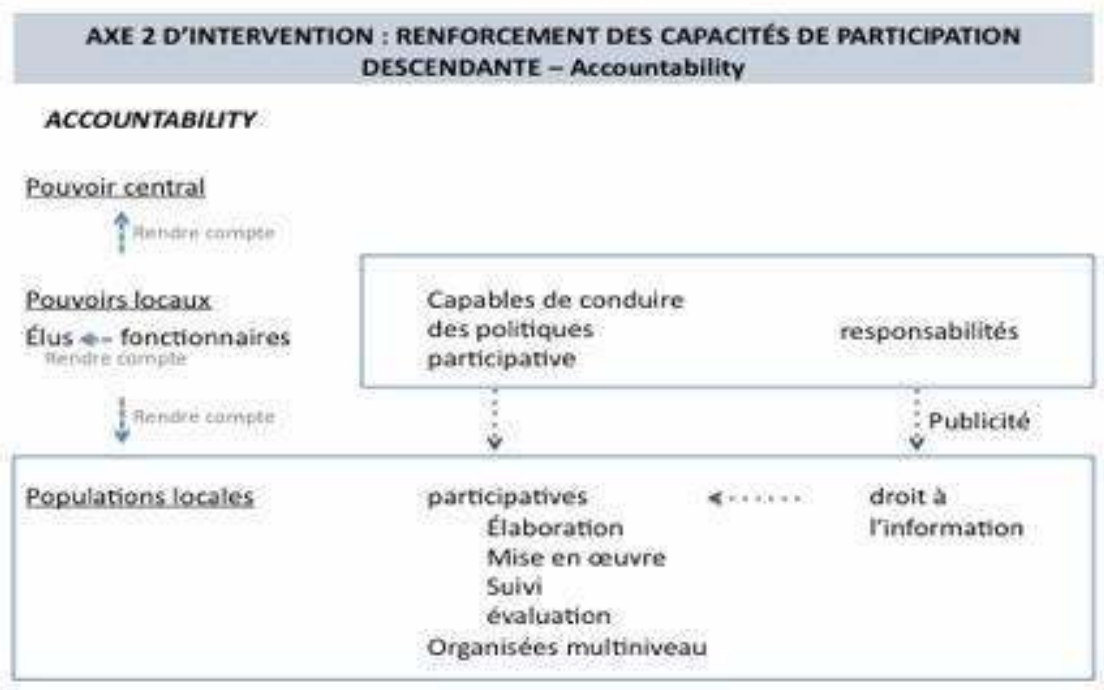

Le postulat théorique du modèle de gouvernance élève ainsi la population au rang d'agent de légitimation à deux points de vue. Premièrement, leur association à la conception et à la mise en œuvre des politiques est supposée conduire à des stratégies mieux adaptées (connaissance des besoins, utilisation et diversification des ressources), ce qui serait susceptible de renforcer la légitimité des pouvoirs locaux à l'égard d'un État central qui, davantage éloigné des réalités locales, serait moins performant. Ensuite, la participation des populations, en ce qu'elle favoriserait le contrôle de la bonne gestion publique, participerait à l'accountability générale de l'État (reddition de comptes) et légitimerait ainsi l'autonomie accordée aux pouvoirs locaux (contre-pouvoirs).

\section{Promouvoir le développement économique et social et lutter contre la pauvreté et les inégalités}

La charte propose d'appuyer un développement équilibré et durable, qui favorise le secteur privé et assure l'inclusion sociale. En matière de délivrance des services publics, la charte insiste sur la nécessité d'assurer l'équité c'est-à-dire que ces services bénéficient au plus grand nombre (couverture, qualité et accès fondés sur les droits sociaux fondamentaux). Elle soutient la participation des acteurs concernés (usagers, prestataires, départements de l'État) et la recherche d'une viabilité institutionnelle, financière et environnementale (compte tenu notamment des spécificités locales). En matière de développement économique territorial, la charte insiste sur une approche multi sectorielle, sur un développement inclusif, favorable au secteur privé (partenariats public-privés, infrastructures, sécurité foncière et accès à des services financiers) et sur l'exploitation des ressources locales. En matière d'environnement, outre qu'il s'agit d'une préoccupation transversale, la charte insiste sur le renforcement de la "participation des autorités locales dans la gestion concertée et durable des ressources naturelles, dans le respect des politiques nationales.» 
Le troisième axe d'intervention se concentre principalement sur la nécessité d'assurer la cohérence des stratégies et l'équilibre entre les différents enjeux liés au développement (qu'ils soient économiques, sociaux et environnementaux). Elle insiste à cette occasion sur le renforcement des capacités et sur les principes de participation multi niveaux. Elle ajoute, en outre, un certain nombre de directives particulières apparentées aux bonnes pratiques (droits de l'Homme et accès le plus large possible aux services sociaux par exemple) $)^{2}$.

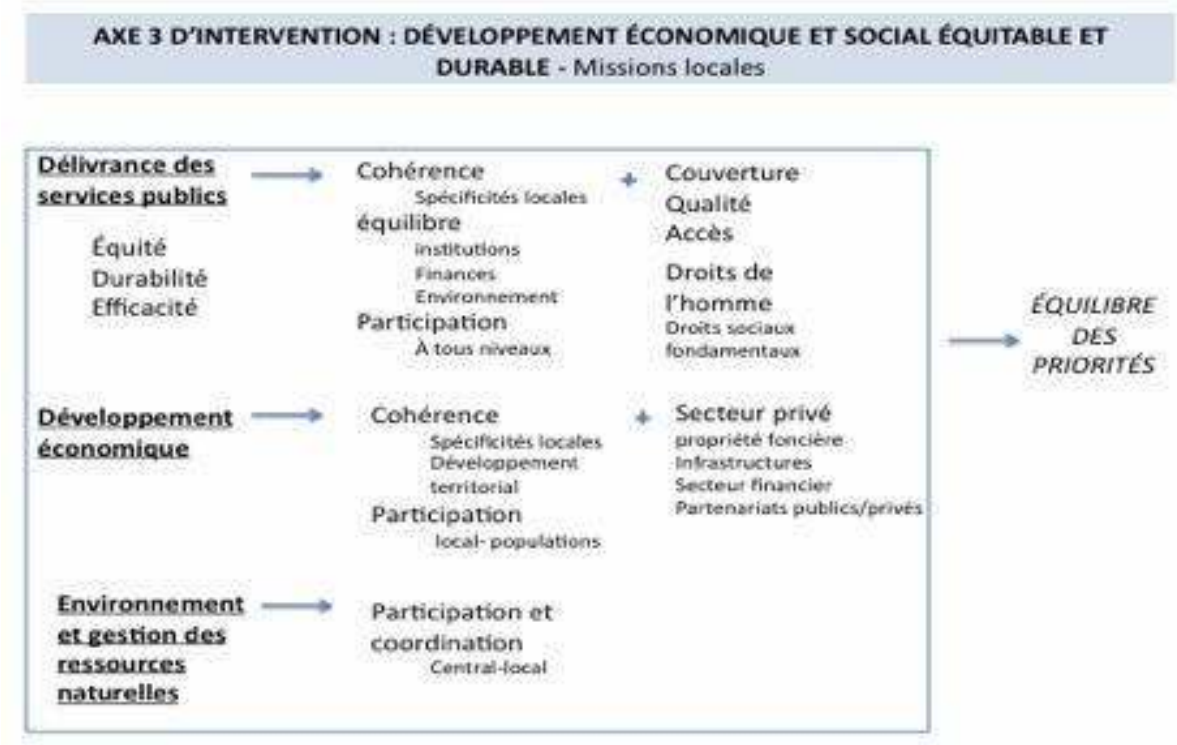

\section{Renforcer la décentralisation fiscale et le financement des autorités locales}

En termes de capacités financières, la charte se propose d'appuyer la diversification des ressources, de telle sorte qu'elles soient constituées par des rentrées fiscales propres, des fonds extérieurs (aide extérieure et marchés financiers) et des transferts nationaux. En terme de bonne gestion, la charte prône l'appui à une politique financière transparente c'est-à-dire informée (participation des acteurs locaux et publicité), motivée (priorisée, équilibrée, réaliste, adaptée aux spécificités locales et cohérente y compris avec les politiques nationales), planifiée, flexible et attentive à la cohésion territoriale. Elle prône également l'association des populations à la mise en œuvre du budget et le renforcement des compétences des agents locaux en matière de gestion des finances publiques. 


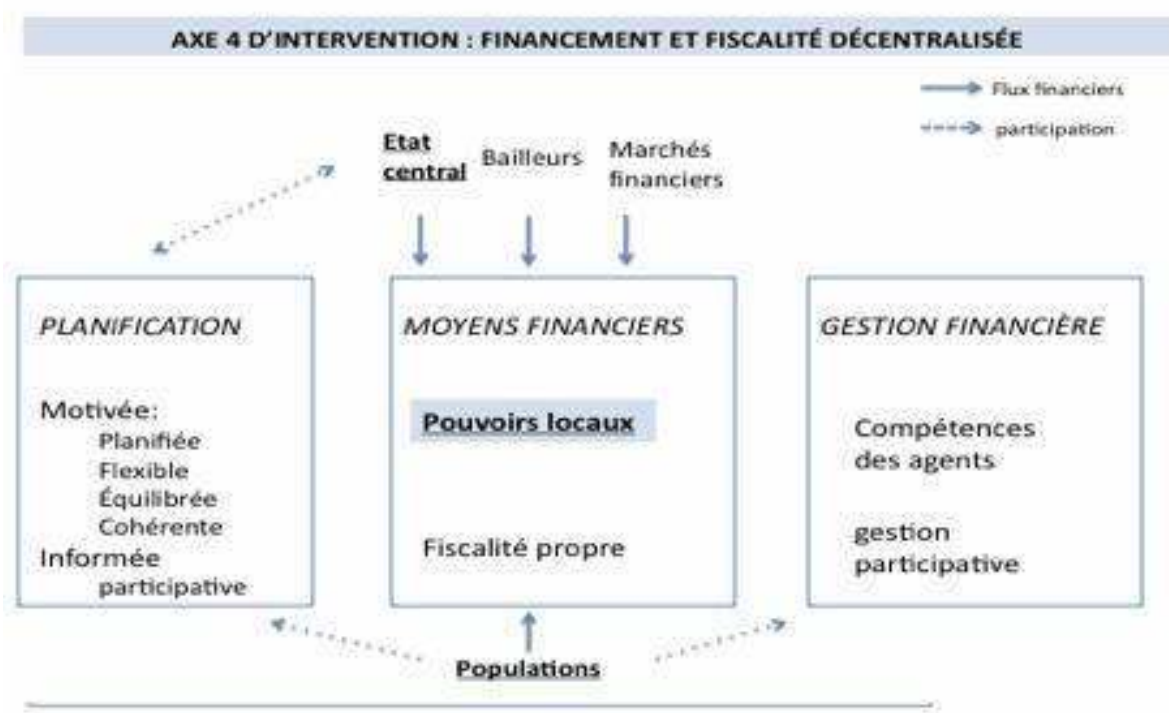
niveaux de lecture. D'une part, elle définit des secteurs d'activités susceptibles de renforcer les processus de décentralisation dans les États partenaires, d'autre part, elle établit des principes directeurs censés encadrer l'action des entités coopérantes, quels que soient les appuis qu'elles apportent aux gouvernements locaux. La charte pourrait ainsi offrir aux ALR européennes le cadre qui leur permettrait non seulement de situer leurs appuis dans une logique de gouvernance, mais également d'identifier, parmi leurs pratiques, celles dont elles pourraient partager l'expérience. Il demeure que les activités de coopération en appui à la gouvernance locale suscitent encore divers questionnements (2).

\section{Questionnements liés à l'opérationnalisation des modèles}

Comme en atteste l'état des réflexions des différents acteurs intéressés par la gouvernance dans les processus de décentralisation, la mise en œuvre de la charte supposera de s'interroger sur la portée exacte des principes promus tels que la participation et le transfert des compétences conformément au principe de subsidiarité par exemple (UN Habitat, 2006; Comité des Régions, 2009b ; UNDP, $2004: 7$; Comité des Régions, 2007, Europaid 2007 ; Vielajus M., $2007: 2$; UNDP, 2007 : 8).

Ainsi, la subsidiarité impose de "définir dans chaque cas, pour chaque compétence, quel est le niveau le plus approprié pour décider et pour agir dans un domaine déterminé» (Polet R., 1997 : 4), de sorte que sa mise en œuvre implique de définir les compétences à transférer, ou le découpage territorial utile pour optimiser les résultats.

L'accountability ascendante suppose de trouver le juste équilibre entre une subordination paralysante des autorités locales, et une "marche vers une décentralisation à l'aveugle » qui nuirait à la cohérence des stratégies nationales sans assurer la bonne conduite des affaires locales (Belloubet-frier N, 2007, § 23; Europaid, 2007; UNDP, 2004 : 10 ; UNDP, 2007 : 16). Un positionnement sur les modalités, du contrôle par l'État central ou de l'exercice d'une tutelle, pourrait alors être requis. 

nécessite de définir les règles qui assureront la professionnalisation de la fonction publique autant que sa dépolitisation et sa stabilité (Vielajus M., 2007:2; UNDP, $2007: 10$; UNDP, $2004: 5-12)$.

L'accountability descendante suppose enfin de s'interroger sur la portée adéquate de l'intéressement des populations aux affaires publiques (dialogue ? referendum ? ...), et sur les moyens mis en œuvre pour en assurer l'efficacité (parer les déficits de légitimité des groupes d'intérêts consultés, sensibiliser les populations aux affaires publiques, renforcer leurs capacités à interagir avec les représentants locaux, à évaluer les besoins et la pertinence ou la qualité de l'action publique ?).

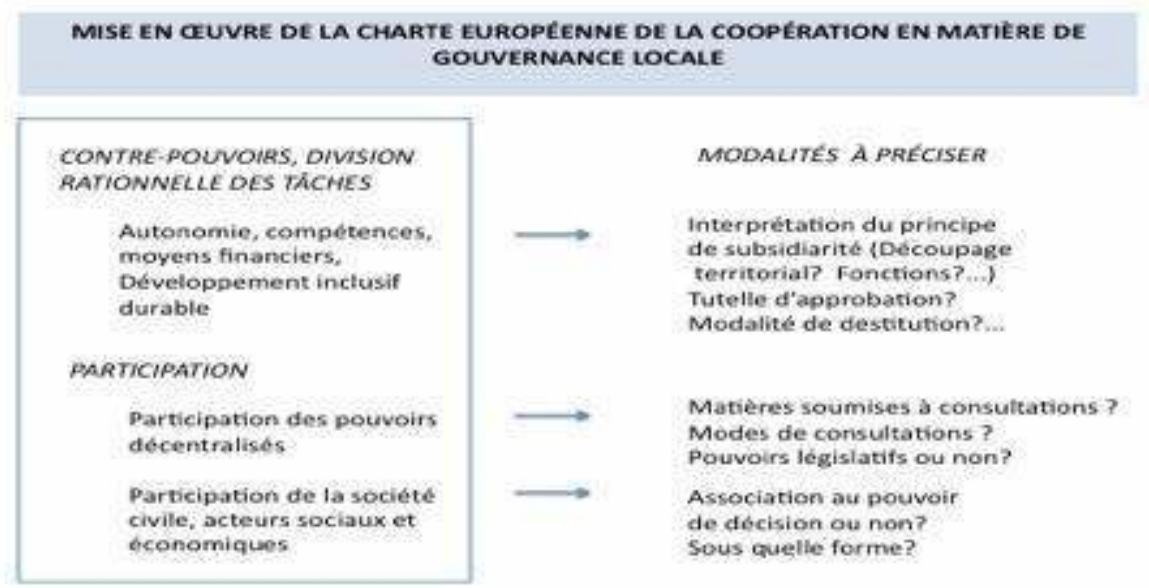

(UN habitat, 2005, pt. A.2\&13 ; UNDP, 2007 : 13-18 ; UNDP, 2004: 14 ; Vielajus M., 2007, pt. 3-4).

Sans prendre directement position sur ces questions, la charte européenne de la coopération en matière d'appui à la gouvernance locale renvoie, en préambule, à divers documents (directives sur la décentralisation et le renforcement des autorités locales adoptées par UN habitat, charte europénne de l'autonomie locale et « autres documents du Conseil de l'Europe »). Ces documents seraient autant d'indices sur l'interprétation à donner aux principes de coopération pour la gouvernance locale dans les pays en développement, en ce qu'ils livrent des réponses partielles aux questions posées cidessus. 


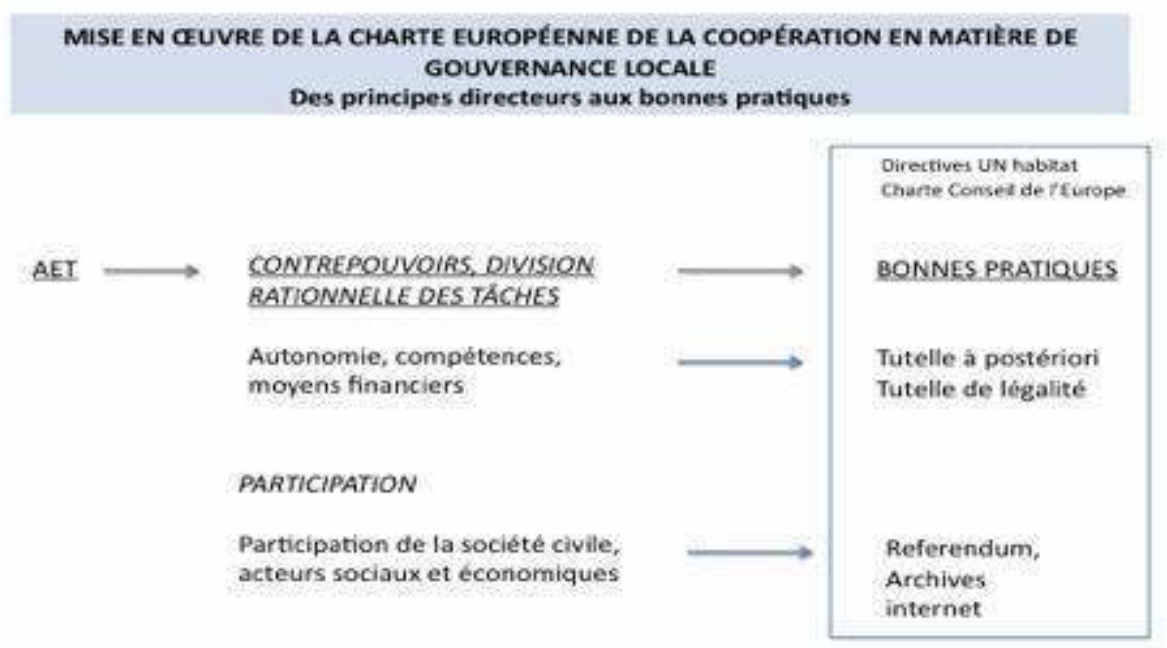

Pour répondre aux défis de l'opérationalisation des stratégies de gouvernance, les entités coopérantes seraient appelées à présenter des bonnes pratiques qui devriendraient autant de modèles de gouvernance. Postuler que les affaires publiques seront efficacement traitées par des contrôles minimaux de l'État central et une participation des groupes d'intérêts aux décisions publiques repose toutefois moins sur des bases empiriques éprouvées ou une connaissance suffisante des mécanismes à l'œuvre dans les pays en développement, que sur une idéologie particulière, qui postule un État moins hiérarchisé, moins régulé (UNDP, 2007 : 26; Nach Mback C., 2003 : 37). Mettre en œuvre les stratégies de gouvernance ne peut en conséquence faire l'économie d'une réflexion suffisante sur l'adéquation des systèmes en présence et sur les orientations politiques qu'ils portent.

\subsection{Enjeux liés aux effets pervers et aux questions de légitimité}

Les études réalisées et l'expérience acquise des processus de décentralisation dans les pays partenaires tendent à mettre en exergue que la disparité des situations nationales, en termes de contraintes et d'opportunités, de schémas culturels, sociaux et historiques, de volonté politique, ou d'équilibre des forces et des intérêts en présence, empêche toute transposition d'un modèle unique (Europaid, 2007, : 35 ; UNDP, 2007).

Sans prétendre à l'exhaustivité, faute de prendre suffisamment en compte ces réalités de terrain, la décentralisation peut conduire à un approfondissement des disparités de développement local, un accroissement de l'instabilité fiscale, ou un affaiblissement de l'État central. À cet égard, les observateurs ont pu observer jusqu'à l'émergence d'« un système de pouvoir politique polycentrique, indépendant et non coordonné qui menace d'étrangler les efforts de développement ». À cela s'ajoute un risque d'accroissement des tensions, que celles-ci concernent les différents groupes sociaux et ethniques, les différents niveaux de pouvoirs, le centre et la périphérie ou encore les collectivités locales elles-mêmes. Enfin, l'expérience suggère qu'une mauvaise conception des processus de participation des populations locales peut accroître les contestations ou, à l'inverse, le désintéressement, voire mener à une polarisation citoyenne. Elle peut enfin conduire à l'affaiblissement des autorités locales, à des concours de pouvoirs et de 
légitimité, à une captation des affaires publiques par les élites, et à une instrumentalisation des réseaux d'association par des groupes d'intérêts particuliers (UNDP, 2004:2-12; UNDP, 2006; UNDP, 2007:6-14; ECPDM, 2008, Czech Republic observation ; Europaid, $2007: 2,8$, 30-35 ; Commission européenne, 2008d:10 ; UN habitat, 2005, pt. A.7 ; Vielajus M., 2007, pt. 4).

Prenant acte de l'ensemble de ces contraintes, la réalisation des objectifs portés par les doctrines de gouvernance suppose flexibilité et progressivité. Ainsi, le referendum comme modalité de participation des populations locales ou l'abandon du contrôle hiérarchique par l'État central au profit d'organes indépendants, considérés comme solutions optimales pour assurer la reddition des comptes dans une logique de contrepouvoirs et de compétition des acteurs, ne sont pas nécessairement posés comme objets d'une mise en œuvre immédiate, mais, le cas échéant, comme objectifs à atteindre.

Ces précautions, indispensables pour éviter les effets pervers induits par les doctrines de gouvernance, laissent ouvertes les questions de légitimité. En effet, la gouvernance postule un mode de régulation international, qui consiste non plus à s'adresser uniquement à l'État central mais à agir, également et directement, sur les acteurs et contre-pouvoirs internes susceptibles de jouer un rôle moteur dans l'appropriation des réformes. La diffusion des modèles de gouvernance auprès de ces acteurs et contrepouvoirs a pour objet de créer des coalitions et enclaves institutionnelles favorables au changement. Ces dernières se feraient alors le relais des modèles diffusés et créeraient par la force de l'exemple, ou par la modification de l'équilibre des forces et intérêts en présence, le consensus ou la volonté politique dont le déficit empêchait, jusque-là, l'appropriation des modèles promus et leur transposition par l'État.

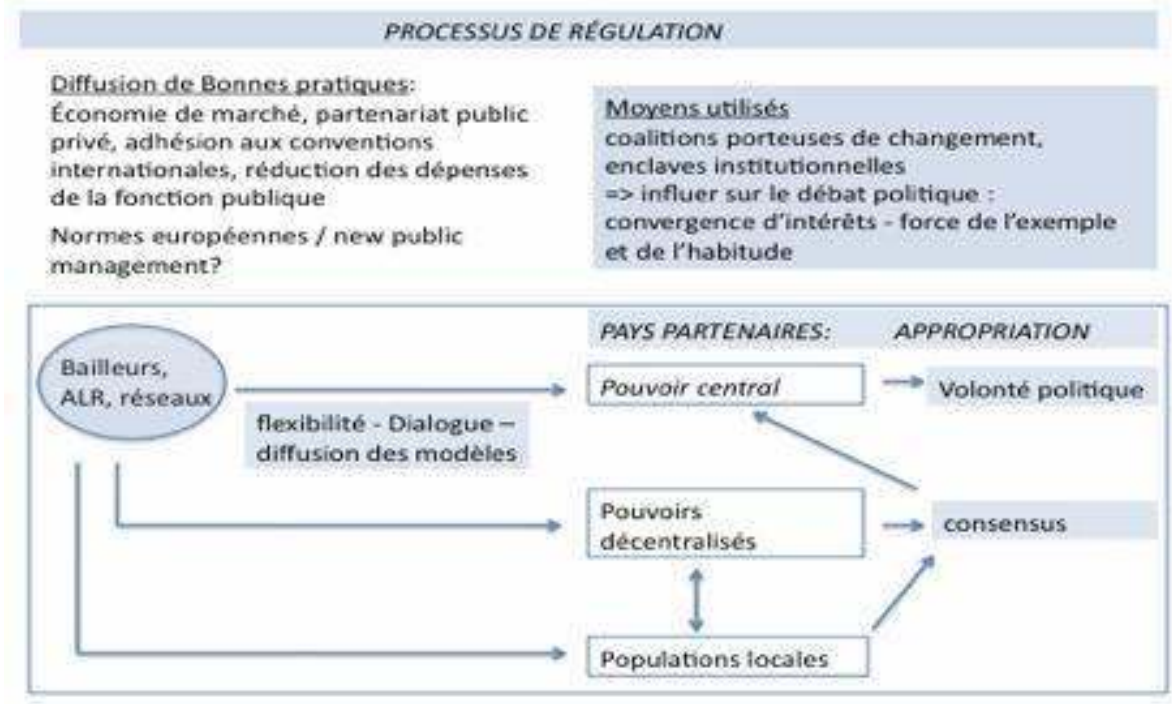

Ces mécanismes de régulation conduisent ainsi les acteurs extérieurs à influer sur les débats politiques internes des États en développement. À ce titre, le référant opéré à des modèles préétablis, en préambule de la charte européenne de la coopération en matière d'appui à la gouvernance locale, pose question. Plaidant un approfondissement de l'autonomie des pouvoirs décentralisés, les modèles diffusés seront vraisemblablement de nature à susciter une convergence d'intérêts entre les bailleurs visant un État moins hiérarchisé et moins régulé et les élus locaux sensibles à l'opportunité de voir s'accroître leur pouvoir de décision. Il reste que, ce faisant, les bailleurs influent sur des choix 
politiques souverains et favorisent l'émergence d'une pression interne pour amener l'État central à adopter un modèle, à l'élaboration duquel il n'a pas participé.

Pour rendre compte du caractère sensible de ces processus de régulation, il suffit de rappeler les difficultés qui entourent, sur la scène intra-européenne, l'élaboration des modèles dont l'exportation est proposée. À titre d'exemple, la charte européenne d'autonomie locale de 1985, présentée comme l'un de ces modèles, a connu une naissance difficile "puisque ce ne seront pas moins de dix-huit années qui s'écouleront entre le lancement des travaux et leur aboutissement» (Levrat N., 2005: 66). Coulé sous forme de traité, certains États, comme la Belgique, ont mis une dizaine d'années supplémentaires à ratifier ce texte, et ce, bien qu'ils aient participé à sa négociation et donc aient « pris soin d'en gommer tous les éléments qui auraient pu être en contradiction avec leur système de partage territorial» (ibid. : 67). L'article 3 bis que le traité de Lisbonne propose d'insérer dans le traité de l'UE: «l'Union respecte l'égalité des États membres devant les traités ainsi que leur identité nationale, inhérente à leurs structures fondamentales politiques et constitutionnelles, $y$ compris en ce qui concerne l'autonomie locale et régionale » nous soulignons. Cela confirme, plus encore, le caractère politique hautement sensible du débat et la prétention des États européens à réserver la question des autonomies locales et régionales dans leur sphère nationale et d'en garder ainsi la maîtrise (ibid. : 68).

La légitimité à voir les États européens exporter les modèles d'autonomie locale dans des États tiers qui n'ont eu ni l'occasion de participer à leur élaboration, ni l'opportunité d'y introduire toutes les nuances utiles à la préservation de leur identité nationale, doit dès lors être posée. La référence en préambule de la charte européenne de coopération en matière de gouvernance locale aux "autres documents du conseil de l'Europe » pose, par ailleurs, les mêmes questions. Les autorités locales et régionales européennes devraient y être d'autant plus sensibles que ces documents véhiculent des modèles qu'elles-mêmes n'ont pas encore pleinement intégré, et qui continuent du reste à faire débat au sein des États européens.

En conclusion, l'exportation des modèles, en ce qu'elle influe sur les choix politiques souverains des États partenaires et en ce qu'elle véhicule des normes qui ne répondent pas nécessairement aux réalités locales et aux identités nationales, devrait à tout le moins prendre toutes les précautions utiles pour discerner les effets pervers qui pourraient être liés à une transposition hâtive des modèles européens et pour préserver la diversité des modèles proposés. C'est seulement à ces conditions que la coopération offrira, aux États centraux et autorités locales des pays en développement, la latitude utile pour identifier les stratégies qui répondraient le mieux à leurs réalités et aspirations (2.2).

\subsection{Perspectives liées aux réalités locales et au maintien de la diversité des modèles}

37 Ayant mis en exergue que « ce qui fonctionne dans un pays nesera pas nécessairement approprié dans un autre» (Europaid, 2007:2) et qu'il n'existe aucun "modèle uniformément transposable» (ibid.: 9), il s'agit de rester attentif aux potentiels effets pervers liés à la transposition des pratiques ou presciptions européennes dans des contextes historiques culturels et politiques parfois fort différents (a), et assurer la diversité des modèles (b). Les ALR européennes sont susceptibles de présenter ici une plus-value toute particulière à l'appui des doctrines de gouvernance. 


\section{Tenir compte des réalités locales} information complète et transparente et susciter le dialogue et la concertation des parties prenantes, représentent des préalables indispensables. Dans un contexte de « learning by doing ", où les bonnes pratiques sont encore à créer, l'analyse éclairée des résultats acquis et des difficultés rencontrées rapportés aux particularités du contexte national et local revêt une importance cruciale. La qualité de ces analyses et leur communication au travers des réseaux d'ALR pourrait contribuer à éviter de proposer des modèles inadéquats et d'en favoriser une appropriation hâtive, alors qu'ils ne sont pas nécessairement directement, voire simplement, transposables. Dans le même ordre d'idées, l'échange d'expériences devrait favoriser l'information rigoureuse sur les problématiques auxquelles les pratiques proposées en exemple étaient censés apporter une réponse, sur les difficultés qui ont été rencontrées lors de leur mise en œuvre éventuelle et sur l'analyse des résultats obtenus. C'est probablement alors que les bailleurs, s'appliquant à eux-mêmes les principes de gouvernance dans un dialogue approfondi avec leurs partenaires, pourront le mieux appuyer l'adoption par ces derniers de modèles adéquats. En résumé, en matière de bonnes pratiques de gouvernance, le « sur mesure " présente de meilleures garanties que le « clé sur porte ».

\section{Préserver la diversité des modèles}

41 Le Comité des Régions souligne la nécessité, au sein de l'Union européenne, de pallier l'un "des risques essentiels de la globalisation", à savoir "le risque d'uniformisation dans nos sociétés » et conclut que «la diversité doit être promue» (Comité des Régions, 2009c, pt. 1). Préserver la diversité des modèles de gestion publique s'avère alors constituer un enjeu, non seulement pour les États en développement, mais également pour les pays européens.

Cela suppose de s'interroger sur les moyens utiles. En effet, si les modèles d'organisation territoriale sont encore largement diversifiés sur le continent européen, de même que les référentiels, intérêts, priorités et aspirations des ALR (N. Levrat, $2005: 37$ ), certains auteurs soulignent qu'il n'est toutefois pas exclu que de nouveaux équilibres se dessinent à l'avenir (ibid. : 51). Divers auteurs constatent, en outre, le mimétisme qui s'opère dans la gestion des collectivités territoriales européennes et l'influence à cet égard des approches entrepreneuriales notamment. Il en va ainsi de «la naturalisation du référentiel du city marketing, la multiplication de structures de partenariats publics-privés ou la transformation de postes de fonctionnaires publics en missions de project managers» (Decroly J-M. et Van 
Criekingen M., 2009 : 4-8). Ce mimétisme reflèterait « la difficulté des autorités urbaines à proposer un modèle de développement urbain qui ne soit pas le décalque des solutions "clés en main" apportées par le monde de l'entreprise " (ibid.: 11). Il y aurait ainsi une propension grandissante «à transposer de manière automatique les outils de la gestion privée» (Senimon M., 2001 : 11), sans prendre le recul suffisant pour évaluer leur pertinence dans un contexte de gestion de la chose publique (ibid.) ou certains de leurs effets que plusieurs études décrivent comme néfastes à la cohésion sociale (Decroly J-M. et Van Criekingen M., 2009 : 5 ; Levrat N., $2005: 50$ ).

Au rang des facteurs susceptibles d'expliquer cette dynamique de mimétisme, les auteurs soulignent l'influence des réseaux d'échanges et des processus de régulation. Ceux-ci, même informels et dépourvus de force contraignante, sont appelés à favoriser l'adoption de référents communs, qui guident le dialogue et tendent à susciter davantage d'uniformisation (UN Habitat, 2006 : 8; Calame, 2008, \$4 ; Bail C., 1996 cité in J. Theys, 2003 \$6; Sennett R., 1990). Certains auteurs constatent ainsi que les textes et recommandations élaborés " tendent à produire une réduction significative des écarts entre les systèmes existants » (Belloubet-frier N, 2007, § 25 ; Polet R., $1997: 4$ ) et soulignent qu'il n'est «pas impropre d'affirmer que des principes communs forcent une dynamique, une démarche où les écarts entre processus de gestion des affaires publiques locales se réduisent. En théorie ce processus porte, à terme, en gestation une harmonisation des différents modèles» (Belloubet-frier N, 2007, § 48). En vue de préserver la diversité, il sera utile de la défendre contre les tendances normatives, c'est-à-dire au premier chef identifier les réseaux d'influences dans la formalisation des standards et des bonnes pratiques.

La complexification des processus de régulation internationaux imposera, au-delà du lobby exercé auprès des institutions disposant d'une compétence normative clairement identifiée (telles le Conseil de l'Europe, l'ONU et l'UE), de distinguer les liens qui se nouent entre les différents acteurs (États, ALR, société civile et organisations internationales), leurs tendances normatives et le poids dont ils disposent dans la définition des bonnes pratiques. En effet, les liens entre les différents lieux de dialogue se multiplient.

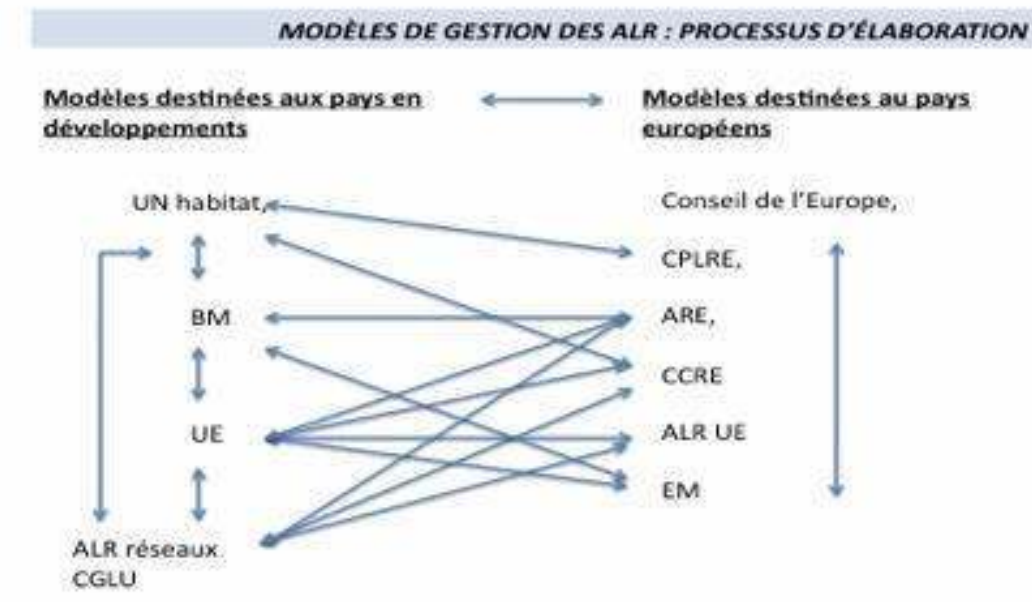

Les influences réciproques entre les différentes sphères de régulation auraient ainsi tendance à s'accroître. Par le biais de représentations en cascade, s'établissent à l'heure actuelle des modèles de gestion de la chose publique, normes qui sans êtres contraignantes deviennent un référent au dialogue, alors même que les acteurs 
directement intéressés n'ont pas nécessairement participé à leur élaboration. La Banque mondiale, lorsqu'elle entend développer les bonnes pratiques en matière de gouvernance locale et de coopération décentralisée, fournit un excellent exemple pour illustrer ce propos. Ainsi, investissant davantage les réseaux existants (World Bank, 2008 et 2009), elle a participé au processus consultatif qui a entouré l'élaboration de la charte européenne de coopération en matière de gouvernance locale. Elle a également créé en 2007 des liens avec l'ARE pour améliorer ses connaissances des structures régionales en Europe et guider ses activités notamment dans les pays appelés à terme à intégrer l'UE³. Elle s'attèle en outre à créer de nouvelles perspectives de régulation et crée en janvier 2009, à cet effet, «l'Alliance des villes ». Elle tisse par ce biais de nouveaux liens avec UN habitat et le CGLU, pour travailler à la coordination des bailleurs et encourager " linvention d'outils et d'approches pratique et le partage de savoir $»^{4}$. Elle est également pressentie, aux côté de l'Alliance des villes et d'UN habitat, pour participer au pôle de travail de Metropolis sur la "gouvernance urbaine intégrée » en 2009-20115. La Banque mondiale, outre son influence sur les modes de régulation des États candidats, renforce ainsi ses liens avec différents acteurs, eux-mêmes susceptibles d'influer sur le contenu de modèles à vocation européenne.

La complexification des processus de régulation et la propension des modèles élaborés à devenir des référents au dialogue entrepris dans les réseaux et enceintes plus formelles de coopération impose, pour garantir la diversité des modèles, que les autres ALR travaillent à identifier les lignes de forces et les moyens d'y être représentées, selon l'impact des normes produites et du poids que les ALR peuvent chacune avoir dans la définition des lignes directrices.

\section{Conclusion}

Les États membres et la Commission européenne se montrent aujourd'hui soucieux d'élaborer des stratégies qui permettent une meilleure complémentarité et coordination des activités avec les ALR. Il reste à renforcer l'information et les données empiriques sur les processus de décentralisation, le cas échéant par l'intermédiaire d'un instrument européen qui permette de rendre compte, de manière exhaustive et pour chacun des pays partenaires, de l'État de la décentralisation, des risques (ex: disparités fiscales, manque de cohésion territoriale, conflits de légitimité, captation par les élites et déficits de légitimité des organes de représentions des populations locales, etc.) et des opportunités (nouvelles législations, institutions, ou expertises locales par exemple). En outre, une approche coordonnée en vue de définir les bonnes pratiques serait également attendue, sachant toutefois que l'un des enjeux serait de veiller à ce que les objectifs de complémentarité et de cohérence ne se conçoivent pas comme le développement d'une stratégie de coopération unique pour l'ensemble des acteurs. La préservation d'une diversité des modèles de gestion publique supposerait alors d'enrichir encore la réflexion sur les outils de régulations que sont les codes de bonnes conduites, la diffusion de bonnes pratiques et la gouvernance multi niveaux notamment. 


\section{BIBLIOGRAPHIE}

Bail C., 1996, "Environmental Governance : Reducing risks in democratic societies". Introduction paper, EEC, Future Studies Unit

Belloubet-frier N, 2007 « Vers un modèle européen d'administration locale ? », Revue française d'administration publique, $1 / 2\left(n^{\circ} 121-122\right)$

CCRE, 2008a, « Vers une approche européenne de la gouvernance locale démocratique, la décentralisation et le développement territorial. Réponse du Conseil des Communes et des Régions d'Europe (CCRE) au papier de discussion de la Commission européenne », Juin 2008

CCRE, 2008b, « Lancement de la nouvelle plateforme des autorités locales et régionales pour le développement », en ligne, consulté le 18 novembre 2009, URL: http://www.cites-uniesfrance.org/IMG/pdf/jed_annonce_plateforme_fr-1.pdf

CGLU, 2008, Réponse de Cités et gouvernements Locaux Unis à la consultation de la Commission européenne, « vers une approche européenne sur la gouvernance démocratique, la décentralisation et le développement territorial », 16 juin 2008.

Calame P., 2008 « L'articulation des échelles de gouvernance : une question omniprésente mais refoulée parce qu'elle heurte de front la théorie politique classique ", Contribution à la charte de la gouvernance locale élaborée par le Ministère français des affaires étrangères ; intervention au séminaire du 28 mars 2008

Charte 2008, charte européenne de la coopération en matière d'appui à la gouvernance locale, Strasbourg, 15-17 novembre 2008, en ligne, consulté le 18 novembre 2009, URL : http:// www.charte-coop-gouvernancelocale.eu/gouvernance/IMG/pdf/ Charte_europeenne_de_la_cooperation_en_matiere_d_appui_a_la_gouvernance_locale-2.pdf CNCD, 2008, « La Charte européenne de la coopération en matière d'appui à la gouvernance locale », http://cncd.diplomatie.gouv.fr/frontoffice/article.asp?aid=343\&lv=2\&menuid=309

Comité des Régions, 2004, « Le rôle des autorités régionales et locales dans le partenariat pour la méditerranée: Comment améliorer et renforcer le partenariat entre les villes et régions du nord et du sud de la méditerranée? », Études CdR E-4/2004, Bruxelles

Comité des Régions, 2007, avis, « La gouvernance dans le consensus européen pour le développement », RELEX-IV-003, 70e session plénière

6 et 7 juin 2007

Comité des Régions, 2009, avis, "Les autorités locales : des acteurs en faveur du développement », 21 et 22 avril 2009, JO, C 200/18 du 25 août 2009

Comité des Régions, 2009b, avis, « Le rôle des collectivités locales et régionales dans le partenariat oriental », RELEX-IV-024, 79e session plénière 21 et 22 avril 2009

Comité des Régions, 2009c avis d'initiative, «Livre blanc du comité des Régions sur la gouvernance à multiniveaux », CONST-IV-020, 80 session plénière 17 et 18 juin 2009

Commission européenne, 1993, « Communication de la Commission du 16 novembre 1993 sur la lutte contre la pauvreté, » $\operatorname{COM}(93) 518$ final, Recueil des communications de la Commission au Conseil 
et au Parlement européen et des Résolutions, déclarations et conclusions correspondantes du Conseil des ministres, Vol 1 « Horizon $2000 »$, mai 1992 - mai 1995

Commission européenne, 2000, Communication de la Commission «La politique de développement de la Communauté européenne », $\operatorname{COM}(2000) 212$ final, 26 avril 2000

Commission européenne, 2003, Communication de la Commission au Conseil, au Parlement européen et Comité économique et social européen, « Gouvernance et développement », COM (2003)615 final, 20 octobre 2003, JO, C 96, 21 avril 2004

Commission européenne, 2005, Communication de la Commission au Conseil, au Parlement européen, au Comité économique et social européen et au Comité des régions, Proposition de déclaration conjointe du Conseil, du Parlement européen et de la Commission, la Politique de Développement de l’Union européenne, « Le Consensus européen », COM(2005) 0311 final Commission européenne, 2006, Communication de la Commission au Conseil, au Parlement européen et au Comité économique et social européen et au Comité des régions, « La gouvernance dans le consensus européen pour le développement. Vers une approche harmonisée au sein de l'Union européenne », $\operatorname{COM}(2006) 421$ final, 30 août 2006

Commission européenne, 2008a, Rapport annuel 2008 sur les politiques de développement et d'aide extérieure de la Communauté européenne et leur mise en œuvre en 2007, COM(2008)379 final, le 23 juin 2008

Commission européenne, 2008b, Communication de la Commission au Conseil, au Parlement européen, au Comité économique et social européen et au Comité des régions, "Les autorités locales: des acteurs en faveur du développement », $\operatorname{COM}(2008) 626$ final, 8 octobre 2008

Commission européenne 2008c, Document accompagnant la Communication de la Commission au Conseil, au Parlement européen, Comité économique et social européen et au Comité des régions, «Les autorités locales: des acteurs en faveur du développement », SEC(2008)2570 final, 8 octobre 2008

Commission européenne 2008d, DG DEV, « Une approche de l'UE pour aborder la gouvernance locale, la décentralisation et le développement territorial -papier de discussion », en ligne, consulté le 18 novembre 2009, http://ec.europa.eu/development/icenter/repository/ Consultation7_issues-paper_2008-04-21_FR.pdf

Conseil UE, 1998, Conclusions du Conseil, « Démocratisation, État de droit, respect des droits de l'homme et bonne gestion des affaires publiques », Conseil développement, 1998, 2141 ème session, Conseil - Développement, 30 novembre 1998, Press:421 Nr: 13461/98

Conseil UE, 2003, Conclusions du Conseil du 17 novembre 2003 sur la « gouvernance et développement », $2541^{\text {ème }}$ session du Conseil - Relations extérieures - Bruxelles, le 17 novembre 2003, Press:321 Nr: 14500/03

Conseil UE, 2008, Conclusions du Conseil sur les autorités locales comme acteurs en faveur du développement pour la réduction de la pauvreté 2902ème session du Conseil affaires générales, le 10 novembre 2008

Decroly J-M, Van Criekingen M., 2009, « Le Plan de Développement International de Bruxelles (PDI). Promesses de développements immobiliers et d'inégalités croissantes? », Brussels Studies $n^{\circ} 25$

Desmet, A. \& Develtere, P., 2002, "Sub-national authorities and developmentco-operation in the OECDDAC member countries", Belgique, Catholic University of Louvain, January 
Dusepulchre, G, 2009, «La gouvernance adressée aux pays en développement : coquille vide ou concept opérationnel? Proposition de clarification à la lumière des documents d'orientation stratégique de la Banque mondiale et de l'Union européenne applicables à l'aide extérieure ", Revue belge de droit international, 1999/2 (sous presse, à paraître)

ECDPM, 2008, EU Member States consultation on the EU Charter on development cooperation in support of local governance, Synthesis Note, avril, en ligne, consulté le 18 novembre 2009, http:// www.charte-coop-gouvernancelocale.eu/gouvernance/spip.php?page=plan\&id_secteur=1

Europaid, 2007, « Appui à la décentralisation et à la gouvernance locale dans les pays tiers », janvier 2007, collection Outils et Méthodes, Document de référence $n^{\circ} 2$

Gaviria, A. A, 2005, Les régions comme acteurs de la coopération entre l'Union européenne et la communauté andine des nations, Institut européen de l'Université de Genève, juin

Leclerc-Olive M., 2005, « Décentralisation et réforme de l'État à l'épreuve des pratiques de coopération », GEMDEV, France-Mali. Regards sur une histoire partagée, Karthala

Levrat N., 2005, L'Europe et ses collectivités territoriales, réflexions sur l'organisation et l'exercice du pouvoir territorial dans un monde globalisé, coll. Cités européennes, PIE Petre Lang, Bruxelles

Local government Association, UK, "Benefits from international co-operation", en ligne, consulté le 18 novembre 2009, http://www.lga.gov.uk/lga/core/page.do?pageId=29185

Metropolis, page d'information, plan de travail 2009-2011, « C3 gouvernance urbaine intégrée », en ligne, consulté le 18 novembre 2009, http://www.flashfutura.com/metropolis/fr/node/1257

Miossec J.-M., 2008, « Le développement local, entre mondialisation et promotion des territoires. De la gouvernance à l'articulation des pouvoirs et des territoires », in A. Diop (dir.), Développement local, gouvernance territoriale, enjeux et perspectives, Karthala, Paris

Nach Mback C., 2003, Démocratisation et décentralisation. Genèse et dynamiques comparés des processus de décentralisation en Afrique subsaharienne, Karthala PDM

OCDE, 2005, Aid Extended by Local and State Governments, Pre-print of the DAC Journal, Volume 6, No. 4

Polet R., 1997, « La relation entre l'administration locale, les régions et le gouvernement central Aperçu européen ", Contribution au séminaire : "The Local Government in Europe", INAP, Madrid, les 4 et 5 novembre

Senimon M., 2001, « Mémorandum, l'évolution des fonctions publiques en Europe », présenté devant le Conseil de l'Europe, octobre

Sennett R., 1990, The conscience of the Eye: the design and social life of cities, New York : Knopf

Theys J., 2003, «La Gouvernance, entre innovation et impuissance », Développement durable et territoires [En ligne], Dossier 2 : Gouvernance locale et Développement Durable, mis en ligne le 01 novembre, Consulté le 29 juillet 2009. URL : http://developpementdurable.revues.org/ index1523.html

UE, 2000, Déclaration commune du Conseil et de la Commission sur la politique de développement de l'Union européenne, du 10 novembre, 2304 ème session, Conseil Développement, Press : 421 Nr 12929/00

UE, 2005, Déclaration conjointe du Conseil et des représentants des gouvernements des États membres réunis au sein du Conseil, du Parlement européen et de la Commission sur la politique de développement de l'Union européenne intitulée « Le consensus européen », novembre ,JO, C 46, 24 février $2006: 1-19$ 
UNDP, 2004, Decentralised Governance for Development: A Combined Practice Note on Decentralisation, Local Governance and Urban/Rural Development, April

UNDP, 2006, “UNDP and governance. Experiences and Lessons Learned”, Management Development and Governance Division, Lessons-Learned Series $n^{\circ} .1,16 / 06 / 2006$

UNDP, 2007 « Vers un Agenda pour la Gouvernance et le Développement Local », DGP-Net Réponse Récapitulative, Décembre 2007, 27 Août - 16 Octobre, en ligne, consulté le 18 novembre 2009, http://www.undp.org/governance/docs/Consolidated-Reply-fr.pdf

UN Habitat, 2005, UN habitat, « Projet de directives sur la décentralisation et le renforcement des autorités locales », HSP/GC/20/7

UN Habitat, 2006, "Local governments and international development co-operation: a European survey on strategies and policies", Best Practices Seville Centre for city-to-city co-operation, May Vielajus M., 2007, IRG (institute for research and debate on governance), Toward a local governance and devlopment agenda. DGP-Net electronic forum - September-october. Mind-Map Synthesis, February 2008, en ligne, consulté le 18 novembre 2009, http://www.institutgouvernance.org/fr/analyse/fiche-analyse-287.html

World Bank, 1992, « Governance and Development », The World Bank

World Bank, 1994, « Governance. The World Bank's Experience », The World Bank

World Bank, 1997 Helping Countries Combat Corruption. The Role of the World Bank, The World Bank, Washington

World Bank,2000, Reforming Public Institutions and Strengthening Governance: A World Bank Strategy, The World Bank, Washington

World Bank, 2002, Reforming Public Institutions and Strengthening Governance: A World Bank Strategy. Implementing update, The World Bank, Washington, april

World Bank, 2003, Reforming Public Institutions and Strengthening Governance: A World Bank Strategy. Implementing update, The World Bank, Washington, april, p. i à xiii.

World Bank, 2007, "Strengthening Bank Group Engagement on Governance and Anti-Corruption.", mars

World Bank, 2007b, "Strengthening World Bank Group Engagement on Governance and Anticorruption : an uptade on the consultations"

World Bank, 2007c, Implementation plan for strengthening world bank group engagement on governance and anticorruption, 2007, en ligne, consulté le 18 novembre 2009, http:// web.worldbank.org/WBSITE/EXTERNAL/TOPICS/ EXTGOVANTICORR/0,,contentMDK:21519459 pagePK:210058 piPK:210062 theSitePK:3035864,00.html

World Bank, 2008, Evaluation de l'aide de la Banque mondiale en faveur de la décentralisation, 1990-2007, résumé analytique, en ligne, consulté le 18 novembre 2009, http:// siteresources.worldbank.org/EXTDECENTR/Resources/decent_es_fr.pdf

World Bank, 2009, Stratégie de la Banque mondiale pour les villes et les collectivités territoriales note conceptuelle et de synthèse, département finances, économie, développement urbain, réseau développement durable, en ligne, consulté le 18 novembre 2009, http:// www.wburbanstrategy.org/urbanstrategy/sites/wburbanstrategy.org/files/WB\%20Urban\% 20Strategy\%20Note\%20\%20--\%20French.pdf 


\section{NOTES}

1. Liste des participants disponible sur le site consacré à la charte : http://www.charte-coopgouvernancelocale.eu/gouvernance/spip.php?article314 ; sur le déroulement du processus et la position des Etats membres: http://www.charte-coop-gouvernancelocale.eu/gouvernance/ spip.php?page=plan\&id_secteur=1; L'ensemble des réponses à la consultation de la commission ayant précédé sa communication «Les autorités locales: des acteurs en faveur du développement", cf. : http://ec.europa.eu/development/how/consultation/index.cfm? action=viewcons\&id=340

2. Il convient de noter que les critères posés au développement d'une stratégie favorable au secteur privé sont communs à l'UE et à la BM. Par contre, faire des droits économiques et sociaux le guide privilégié pour juger de la couverture, la qualité et l'accessibilité des services publics traduit une conception davantage européenne de la gouvernance. Ici l'Union européenne se rapproche des principes promus par le PNUD, également attachés aux droits fondamentaux.

3. http://www.aer.eu/fr/themes-majeurs/governance/aerworld-bank-research-project.html

4. L'alliance pour les villes, Charte, 23 janvier 2009, http://www.citiesalliance.org/doc/charter/ Charter\%20-\%2023\%20January\%202009\%20-\%20French.pdf

5. Metropolis, page d'information, plan de travail 2009-2011, «C3 gouvernance urbaine intégrée », http://www.flashfutura.com/metropolis/fr/node/1257

\section{RÉSUMÉS}

L'Union européenne connaît une nouvelle dynamique qui, s'intéressant à l'investissement croissant des autorités locales dans les activités de coopération au développement, tend à l'encadrer davantage. L'article met en exergue que, formalisée notamment dans «la charte européenne de la coopération en matière d'appui à la gouvernance locale ", cette dynamique permet aux bailleurs traditionnels de trouver de nouveaux relais à l'appui de leurs stratégies visant à promouvoir la gouvernance et la décentralisation, mais laisse certaines questions sans réponse

This paper tackles the new focus of the European Union on local authorities as actors for development. In putting it in its context, namely the strategies which promote governance and decentralization, the purpose of this article is two-fold. On the one hand, it seeks to highlight the assets of this new interest. On the other, it attempts to draw out some of the questions still pending

\section{INDEX}

Keywords : governance, local governance, decentralization, development cooperation, accountability, transparency, empowerment

Mots-clés : gouvernance, gouvernance locale, pouvoirs locaux, autorités locales et régionales, coopération au développement, coopération décentralisée 


\section{AUTEUR}

\section{GAËLLE DUSEPULCHRE}

Gaëlle Dusepulchre est docteur en sciences juridiques et chargée de recherches à l'Institut d'Etudes Européennes/Université Libre de Bruxelles, Belgique. Elle travaille notamment sur les relations extérieures et la politique de coopération au développement de l'Union européenne en lien avec les droits de l'homme et les stratégies de gouvernance. gaelle.dusepulchre@ulb.ac.be Cette contribution est réalisée dans le cadre d'une recherche post-doctorale : Prospective Research For Brussels 2007. 